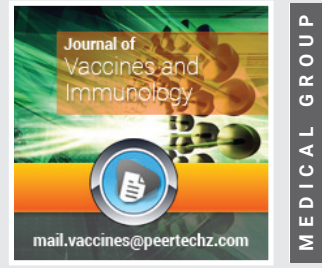

\section{An alternative strategy for studying emerging atypical porcine pestivirus}

\author{
Xujiao Ren ${ }^{1,2}$, Xueyan Liu' ${ }^{1,2}$, Jianglong $\mathrm{Li}^{1,2}$, Huanchun Chen ${ }^{1-3}$, \\ Xiangmin $\mathrm{Li}^{1-3}$ and Ping Qian ${ }^{1-3 *}$
}

'State Key Laboratory of Agricultural Microbiology, Huazhong Agricultural University, Wuhan 430070,

Hubei, China

${ }^{2}$ Laboratory of Animal Virology, College of Veterinary Medicine, Huazhong Agricultural University,

Wuhan 430070, Hubei, China

${ }^{3}$ Key Laboratory of Preventive Veterinary Medicine in Hubei Province, The Cooperative Innovation

Center for Sustainable Pig Production, Wuhan, Hubei, 430070, China
Received: 23 September, 2020

Accepted: 30 September, 2020

Published: 01 October, 2020

*Corresponding author: Ping Qian, State Key Laboratory of Agricultural Microbiology, Huazhong Agricultural University, Wuhan 430070, Hubei, China, Tel: +86 27 8728 2608; Fax: +86 278728 2608; E-mail: qianp@mail.hzau.edu.cn

Keywords: Pestivirus; APPV; Congenital tremor; Vaccines; Reverse genetics system

https://www.peertechz.com

Check for updates

\begin{abstract}
Atypical Porcine Pestivirus (APPV) is an emerging agent that belongs to the genus Pestivirus of the family Flaviviridae and causes Congenital Tremor (CT) in newborn piglets. Piglets with CT are mainly characterized by rhythmic tremor in the limbs and head, complicated by ataxia. Affected animals often die due to insufficient sucking with a mortality rate of $10 \%-30 \%$. Histopathological findings of such piglets are mainly characterized by increased vacuoles in the white matter of the cerebellum, hypomyelination of the spinal cord, and microglial proliferation. APPV has been widely spread around the world since it was first reported in 2015 , bringing huge economic losses to the pig industry. However, as a newly discovered virus, no vaccine is currently available to prevent and control APPV infection. In addition, the difficulties in APPV isolation and its high genetic variability severely hamper the development of APPV vaccines. Here, we propose an alternative strategy, the reverse genetics system, may be a prospective platform to address the issues with APPV vaccine design.
\end{abstract}

\section{Introduction}

Congenital Tremor (CT) of piglets, also known as "shaker pig disease" or "dancing pig disease", is a neurogenic disease that occurs in newborn piglets. At present, CT has been classified into type A and type B according to whether the Central Nervous System (CNS) has histopathological lesions. CT type A is mainly manifested by vacuolization and hypomyelination in the cerebellar and spinal cord, while CT type B has no significant pathological changes in the CNS. Based on the distinct causative factors, CT type A is further categorized into five subtypes (types A I-V). Recent studies have shown that the newly emerging virus, Atypical Porcine Pestivirus (APPV), may be associated with CT type A-II $[1,2]$.

Currently, accumulating evidence indicates that APPVinfected CT type A-II has developed into a global disease, thus posing great challenges to pig production. Although numerous studies have been carried out on APPV in the past few years [3-7], including epidemiology and genetic evolution, there are relatively few reports on its pathogenesis, immune response, transmission route, and particularly vaccine design, the most important aspects of disease prevention. Additionally, the virus is difficult to isolate and passage in cell culture, which significantly hinders APPV research, and leads to a need of finding new and alternative approaches to study the virus. Thus, a comprehensive summary of the latest findings on APPV will contribute to further research on APPV and provide new strategies for the design of APPV vaccines.

\section{Obstacles to APPV vaccine development}

Vaccination is an effective measure to prevent infectious diseases. However, there is currently no commercial vaccine for the prevention and control of APPV infection. Since the discovery of APPV, numerous efforts have been made to isolate 
and culture the virus on multiple cell lines (MDBK, MDCK, PK15, and Vero, etc.), but all have failed [3]. Notably, recent studies have shown that APPV from positive serum samples could be amplified on several cell lines, even though the virus titers were low $[4,8]$, bringing strong prospects for APPV isolation. The inability to effectively propagate the virus in cell cultures substantially hampers the development of APPV vaccines. However, novel vaccine designs provide new strategies for the prevention and control of APPV. A subunit vaccine based on APPV E2 glycoprotein has been developed in our laboratory [9], and the results showed that the subunit vaccine could induce a Th2-type immune response in mice. The immunogenicity of the subunit vaccine must be further evaluated in swine herds.

\section{Reverse genetics system may be a promising platform for APPV research}

Reverse genetics is a methodology opposite to classical genetics, which studies the structure and function of biological genes by conducting site-directed mutation, insertion, deletion, and substitution on the basis of obtaining biological gene information. Since the successful rescue of the first RNA virus $Q \beta$ phage in 1978 [10], great progress has been made in molecular biology research of various RNA viruses. In 1981, the first full length cDNA clone of a eukaryotic positive stranded RNA virus, the poliovirus, was constructed successfully [11], which opened the new era of reverse genetics research. The pestiviruses genome is a positive-sense single-stranded
RNA, which can be directly used as messenger RNA (mRNA) to produce enzymes required for RNA replication. Thus, it is easy to manipulate the viral genome at the level of cDNA. Construction of infectious clones of RNA viruses provides robust reverse genetic tools for pestiviruses (Figure 1), and may be a feasible solution for further research on APPV.

Based on the infectious clone, the deletion, site-directed mutation, and substitution of the viral genome can reveal the roles of various regulatory sequences and viral proteins in the viral life cycle and pathogenesis [12]. In addition, the infectious clone can also serve as a useful tool for antiviral assays via the insertion of exogenous sequences [13].The establishment of the reverse genetics system provides a new approach for the development of pestivirus vaccines, including live attenuated vaccines and chimeric vaccines [14,15]. Moreover, infectious clones can be used as seeds for existing live attenuated vaccines to reduce production batch differences and ensure vaccine stability and safety.

\section{Conclusions and perspectives}

Pestiviruses are one of the most important pathogens of infectious diseases in the breeding industry, resulting in considerable economic losses worldwide. The high genetic variability of pestiviruses is the key to triggering epidemics. Although extensive research has been conducted on APPV, in view of the wide distribution and genetic variability of

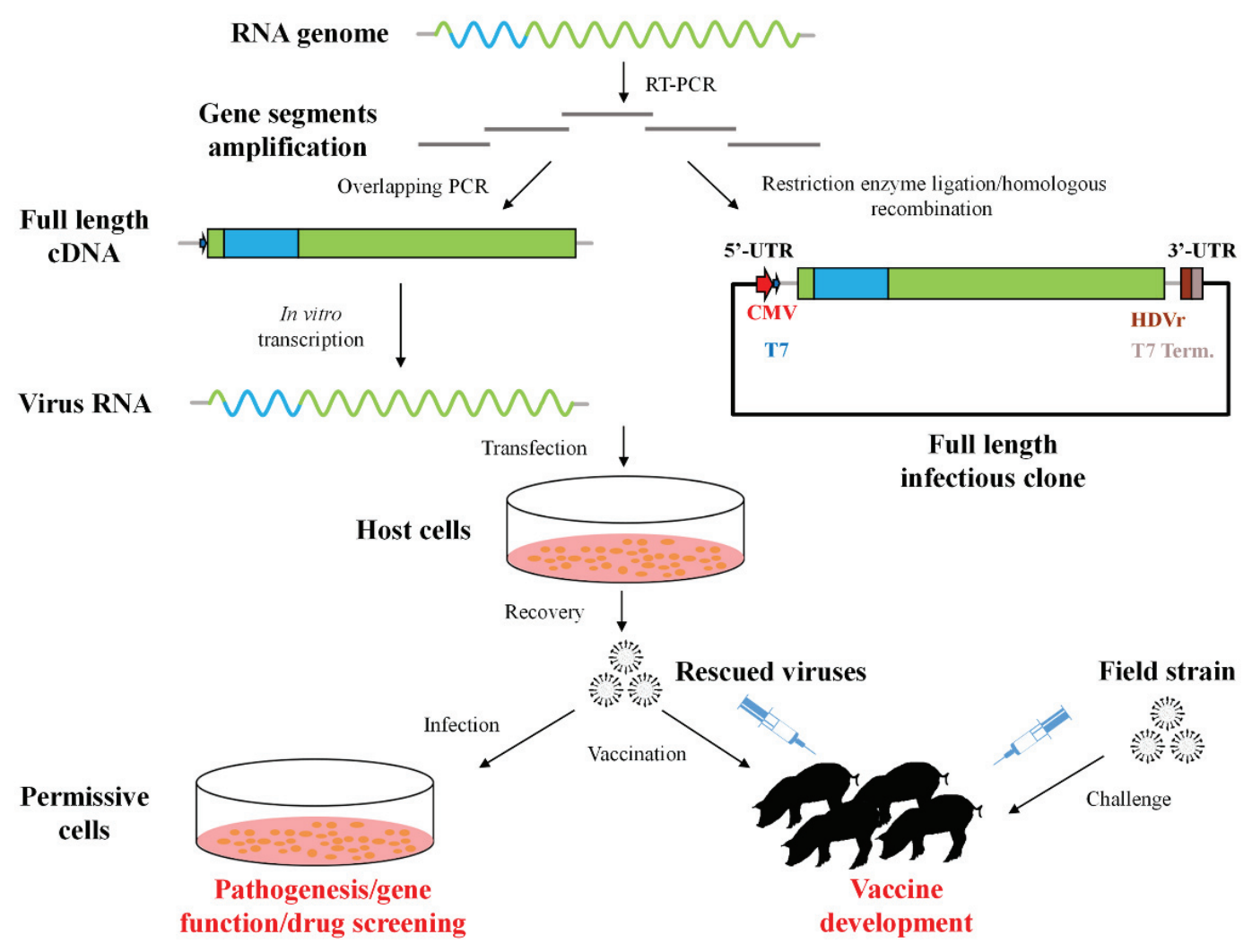

Figure 1: General strategy for the construction of reverse genetics system of pestivirus and its application. The full length cDNA or infectious clone of pestivirus is constructed via overlapping PCR and restriction enzyme ligation or homologous recombination. After purification of the constructs, infectious viruses are rescued either via transfection of virus RNA obtained by in vitro transcription or via direct transfection of infectious clone when using a bacteriophage promoter or a eukaryotic promoter. Rescued viruses can be used for further research, including pathogenesis, gene function, drug screening, and vaccine development, etc.

Citation: Ren X, Liu X, Li J, Chen H, Qian P, et al. (2020) An alternative strategy for studying emerging atypical porcine pestivirus. J Vaccines Immunol 6(1): 022-025. DOI: https://dx.doi.org/10.17352/jvi.000032 
APPV in different countries, it is necessary to conduct an in-depth study on its etiology, epidemiology, transmission routes, pathogenesis, diagnosis, and preventive strategies. In particular, an epidemiological surveillance system for APPV should be established to explore the genetic and evolutionary relationships of the virus.

Previous studies have reported that APPV can be found in semen from commercial boar studs [16], hence, the crucial role of semen in the dissemination of APPV requires further investigation. In addition, recent studies have revealed the presence of APPV in wild boars, suggesting that the wild boars may be a critical virus reservoir for APPV [17-19]. Further investigation is required to elucidate the role of wild boars in the epidemiology of APPV. So far, few data are available regarding the immune response against APPV. Although IFN- $\alpha$ was detected in naturally infected piglets [20] and the humoral immune response against APPV has been described [21] in two independent studies, the important role of the innate and adaptive immune response to APPV still needs further study.

As a newly discovered virus, no commercial vaccine is currently available against APPV infection. Furthermore, the virus is difficult to isolate and passage in cell cultures, resulting in the deficiency of live virus materials, which severely hampers the intensive study of APPV. The reverse genetics system is an effective tool for molecular biology research and development of new vaccines for RNA viruses. Multiple reverse genetics systems of pestiviruses have been developed to study their pathogenesis, gene function, and vaccine design, which may become a prospective strategy to address the issues with APPV vaccine design. Although a reverse genetics system for APPV has been successfully established in a recent study [22], the titer of the rescued virus is low, and the biological characteristics including virulence and pathogenicity of the rescued virus have not been investigated in depth. Similar to other flaviviruses, the cDNA clone of APPV is unstable in host bacteria, which makes its genome prone to mutation. Several approaches could be employed to overcome this problem, including in vitro ligation / transcription [22,23], intron insertion [24], reduction of bacterial promoter activity [25,26], and use of low-copy-number plasmids [27,28]. Furthermore, greater efforts are required to ensure vaccine stability and safety, and to improve the efficiency of the reverse genetics system for APPV, to offer a solid foundation for studying the replication, assembly, invasion mechanism, and pathogenesis of APPV. Meanwhile, animal models of APPV infection can be constructed by using rescued virus or APPV-positive serum to further facilitate the development of APPV vaccines, such as E2 protein-based subunit vaccines. Continued surveillance of epidemiology for APPV and intensive vaccine development will help eradicate the disease and maintain a healthy pig production environment.

\section{Funding}

This work was supported by the National Program on Key Research Project of China (2018YFD0500801, 2018YFD0500204), the Fundamental Research Funds for the Central Universities (2662016PY003).

\section{References}

1. de Groof A, Deijs M, Guelen L, van Grinsven L, van Os-Galdos L, et al. (2016) Atypical Porcine Pestivirus: A Possible Cause of Congenital Tremor Type A-II in Newborn Piglets. Viruses 8: 271. Link: https://bit.ly/33d1bE0

2. Postel A, Hansmann F, Baechlein C, Fischer N, Alawi M, et al. (2016) Presence of atypical porcine pestivirus (APPV) genomes in newborn piglets correlates with congenital tremor. Sci Rep 6: 27735. Link: https://bit.ly/2G2oJmx

3. Hause BM, Collin EA, Peddireddi L, Yuan F, Chen Z, et al. (2015) Discovery of a novel putative atypical porcine pestivirus in pigs in the USA. J Gen Virol 96 : 2994-2998. Link: https://bit.ly/2Gmk7rb

4. Beer M, Wernike K, Dräger C, Hoper D, Pohlmann A, et al. (2017) High Prevalence of Highly Variable Atypical Porcine Pestiviruses Found in Germany. Transbound Emerg Dis 64: e22-e26. Link: https://bit.ly/2S9BtKe

5. Zhang K, Wu K, Liu J, Ge S, Xiao Y, et al. (2017) Identification of atypical porcine pestivirus infection in swine herds in China. Transbound Emerg Dis 64: 1020-1023. Link: https://bit.ly/349MMYG

6. Pan S, Yan Y, Shi K, Wang M, Mou C, et al. (2019) Molecular characterization of two novel atypical porcine pestivirus (APPV) strains from piglets with congenital tremor in China. Transbound Emerg Dis 66: 35-42. Link: https://bit.ly/3ik1LUS

7. Yan XL, Li YY, He LL, Wu JL, Tang XY, et al. (2019) 12 novel atypical porcine pestivirus genomes from neonatal piglets with congenital tremors: A newly emerging branch and high prevalence in China. Virology 533: 50-58. Link: https://bit.ly/3ikz1v3

8. Schwarz L, Riedel C, Högler S, Sinn LJ, Voglmayr T, et al. (2017) Congenita infection with atypical porcine pestivirus (APPV) is associated with disease and viral persistence. Vet Res 48: 1. Link: https://bit.ly/33eP6yf

9. Zhang $\mathrm{H}$, Wen $\mathrm{W}$, Hao $\mathrm{G}$, Chen $\mathrm{H}$, Qian $\mathrm{P}$, et al. (2018) A Subunit Vaccine Based on E2 Protein of Atypical Porcine Pestivirus Induces Th2-type Immune Response in Mice. Viruses 10. Link: https://bit.ly/3kYAdGj

10. Taniguchi T, Palmieri M, Weissmann C (1978) QB DNA-containing hybrid plasmids giving rise to QB phage formation in the bacterial host. Nature 274 223-228. Link: https://bit.ly/2SdzaGk

11. Racaniello VR, Baltimore D (1981) Cloned poliovirus complementary DNA is infectious in mammalian cells. Science 214: 916-919. Link: https://bit.ly/2G3ItpX

12. Li Y, Xie L, Zhang L, Wang X, Li C, et al. (2018) The E2 glycoprotein is necessary but not sufficient for the adaptation of classical swine fever virus lapinized vaccine C-strain to the rabbit. Virology 519: 197-206. Link: https://bit.ly/3iiOayV

13. Shen L, Li Y, Chen J, Li C, Huang J, et al. (2014) Generation of a recombinant classical swine fever virus stably expressing the firefly luciferase gene for quantitative antiviral assay. Antiviral Res 109: 15-21. Link: https://bit.ly/3ibrJtz

14. Lim SI, Choe S, Kim KS, Jeoung HY, Cha RM, et al. (2019) Assessment of the efficacy of an attenuated live marker classical swine fever vaccine (Flc-LOM-BE(rns)) in pregnant sows. Vaccine 37: 3598-3604. Link: https://bit.ly/34eJovu

15. Yang Z, Wu R, Li RW, Li L, Xiong Z, et al. (2012) Chimeric classical swine fever (CSF)-Japanese encephalitis (JE) viral replicon as a non-transmissible vaccine candidate against CSF and JE infections. Virus Res 165: 61-70. Link: https://bit.ly/3n60PqX

16. Gatto IRH, Arruda PH, Visek CA, Victoria JG, Patterson AR, et al. (2018) Detection of atypical porcine pestivirus in semen from commercial boar studs in the United States. Transbound Emerg Dis 65: e339-e343. Link: https://bit.ly/2Sb4TI3 
17. Cagatay GN, Antos A, Meyer D, Maistrelli C, Keuling O, et al. (2018) Frequent infection of wild boar with atypical porcine pestivirus (APPV). Transbound Emerg Dis 65: 1087-1093. Link: https://bit.ly/3ihnkFs

18. Sozzi E, Salogni C, Lelli D, Barbieri I, Moreno A, et al. (2019) Molecular Survey and Phylogenetic Analysis of Atypical Porcine Pestivirus (APPV) Identified in Swine and Wild Boar from Northern Italy. Viruses 11. Link: https://bit.ly/3n5g3w3

19. Choe S, Park GN, Cha RM, Hyun BH, Park BK, et al. (2020) Prevalence and Genetic Diversity of Atypical Porcine Pestivirus (APPV) Detected in South Korean Wild Boars. Viruses 12: 680. Link: https://bit.ly/3I3jYYL

20. Muñoz-González S, Canturri A, Pérez-Simó M, Bohórquez JA, Rosell $R$, et al. (2017) First report of the novel atypical porcine pestivirus in Spain and a retrospective study. Transbound Emerg Dis 64: 1645-1649. Link: https://bit.ly/2ShTfLn

21. Cagatay GN, Meyer D, Wendt M, Becher P, Postel A (2019) Characterization of the Humoral Immune Response Induced after Infection with Atypical Porcine Pestivirus (APPV). Viruses 11: 880. Link: https://bit.ly/33fg1Kr

22. Liu J, Ren X, Li H, Yu X, Zhao B, et al. (2020) Development of the reverse genetics system for emerging atypical porcine pestivirus using in vitro and intracellular transcription systems. Virus Res 283: 197975. Link: https://bit.ly/2HI2q62
23. Sumiyoshi $\mathrm{H}$, Hoke $\mathrm{CH}$, Trent DW (1992) Infectious Japanese encephalitis virus RNA can be synthesized from in vitro-ligated cDNA templates. J Virol 66: 5425-5431. Link: https://bit.ly/3jjDwro

24. Tsetsarkin KA, Kenney H, Chen R, Liu G, Manukyan H, et al. (2016) A FullLength Infectious cDNA Clone of Zika Virus from the 2015 Epidemic in Brazi as a Genetic Platform for Studies of Virus-Host Interactions and Vaccine Development. mBio 7. Link: https://bit.ly/3jh3g7x

25. Pu SY, Wu RH, Yang CC, Jao TM, Tsai MH, et al. (2011) Successful propagation of flavivirus infectious cDNAs by a novel method to reduce the cryptic bacterial promoter activity of virus genomes. J Virol 85: 2927-2941. Link: https://bit.ly/33hsvBj

26. Pu SY, Wu RH, Tsai MH, Yang CC, Chang CM, et al. (2014) A novel approach to propagate flavivirus infectious cDNA clones in bacteria by introducing tandem repeat sequences upstream of virus genome. J Gen Virol 95: 1493-1503. Link: https://bit.ly/3I58aVX

27. Zheng $\mathrm{H}$, Zheng $\mathrm{X}$, Tong W, Liu F, Liang C, et al. (2017) A simple method for developing an infectious cDNA clone of Japanese encephalitis virus. Virus Genes 53: 4-14. Link: https://bit.ly/348XPkR

28. Ávila-Pérez G, Park JG, Nogales A, Almazán F, Martínez-Sobrido L (2019) Rescue of Recombinant Zika Virus from a Bacterial Artificial Chromosome cDNA Clone. J Vis Exp. Link:https://bit.ly/33fgr3t

\section{Discover a bigger Impact and Visibility of your article publication with} Peertechz Publications

Copyright: ( $) 2020$ Ren X, et al. This is an open-access article distributed under the terms of the Creative Commons Attribution License, which permits unrestricted use distribution, and reproduction in any medium, provided the original author and source are credited.

Citation: Ren X, Liu X, Li J, Chen H, Qian P, et al. (2020) An alternative strategy for studying emerging atypical porcine pestivirus. J Vaccines Immunol 6(1): 022-025. DOI: https://dx.doi.org/10.17352/jvi.000032 